\title{
LA APRECIACIÓN Y LA PRODUCCIÓN DEL CHISTE EN E/L2. UN SALTO COGNITIVO Y METAPRAGMÁTICO*
}

\author{
JOKE APPRECIATION AND PRODUCTION IN L2 SPANISH. \\ A COGNITIVE AND METAPRAGMATIC LEAP
}

\author{
Esther Linares Bernabéu \\ Universidad de Alicante. \\ Grupo de investigación GRIALE \\ Esther.linares@ua.es
}

Recibido: 04/09/2017

Aceptado: 17/10/2017

\begin{abstract}
Resumen
Este trabajo analiza la competencia humorística de estudiantes de español como segunda lengua, a través de los parámetros de la apreciación y la producción del chiste. En concreto, el estudio ha contado con la participación de 71 adolescentes extranjeros, que se encontraban en España realizando sus estudios de Educación Secundaria Obligatoria o Bachillerato durante el curso académico 2016-2017. Para alcanzar el objetivo principal de esta investigación, se llevó a cabo un análisis cuantitativo y cualitativo de los datos recogidos por medio de un cuestionario y de una plantilla de observación. En el estudio observamos cómo influyen variables como el grado de inmersión lingüística, el tiempo de estancia en España, el nivel de
\end{abstract}

\begin{abstract}
This paper aims at analysing humour competence in learners who study Spanish as a second language, through the parameters of joke's appreciation and production. More precisely, the study has counted with the participation of 71 foreign teenagers, who were studying either Compulsory Secondary Education or Upper Secondary Education during the academic year 2016-2017 in Spain. In order to achieve our purpose, data collected with a questionnaire and an observation schedule were analysed quantitatively and qualitatively. In this study, we examine how factors such as language immersion, the length of stay in Spain, language level, and the learner's gender and age affect the development of humour and metapragmatic
\end{abstract}

\footnotetext{
* La investigación recogida en el presente artículo ha sido posible gracias al contrato predoctoral financiado por la Conselleria d'Educació, Investigació, Cultura i Esport de la Generalitat Valenciana a través del Proyecto de investigación Humor de género: Observatorio de la identidad de mujeres y hombres a través del humor (PROMETEO/2016/052 IP Leonor Ruiz Gurillo).
}

Para citar este artículo / To cite this article: Linares Bernabéu, Esther (2017). La apreciación y la producción del chiste en E/L2. Un salto cognitivo y metapragmático. ELUA, 31: 211-231. doi: 10.14198/ELUA2017.31.11

Enlace / Link: http://dx.doi.org/10.14198/ELUA2017.31.11 
lengua, o el género y la edad del estudiante en la evolución de las competencias humorística y metapragmática. Los datos obtenidos en esta investigación serán fundamentales para poder elaborar recursos didácticos adaptados a las capacidades y preferencias de nuestro alumnado.

PALABRAS CLAVE: humor en E/L2, chiste, apreciación, producción, metapragmática competences. Data obtained in this research will be crucial for future elaboration of didactic material, in which we consider our students' aptitudes and preferences.

KEYWORDS: Humor in L2 Spanish, joke, appreciation, production, metapragmatics

\section{INTRODUCCIÓN}

El sentido del humor es un rasgo propio de la identidad del ser humano y, sin duda, es una de nuestras mayores virtudes. El humor ejerce una función social en la creación de lazos de apego y complicidad entre los hablantes (cfr. Boxer y Cortes-Conde, 1997; Hay, 2000; Coates, 2003), por lo que, a la vez, fomenta los sentimientos de compañerismo y adhesión al grupo. Ahora bien, el uso del humor en la conversación coloquial, que $a$ priori puede parecer natural y espontáneo, supone para el hablante no nativo una profunda reflexión sobre la función del lenguaje en el determinado contexto humorístico. Es decir, conlleva la activación de sus habilidades metapragmáticas (cfr. Verschueren, 2000; Ruiz Gurillo, 2016), las cuales, si no han sido correctamente fomentadas, no serán suficientes para lograr inferir el humor del enunciado. Esta sería, pues, la principal razón por la que consideramos de vital importancia desarrollar las competencias humorísticas y metapragmáticas en el aula de español como Segunda Lengua (en adelante, E/L2) desde niveles iniciales. Si trabajamos con muestras humorísticas dentro de nuestra programación didáctica, estaremos fomentando la seguridad y confianza de nuestro alumnado internacional para entablar conversaciones más cómodamente, así como contribuyendo a su integración dentro de la comunidad nativa de hablantes. Así pues, compartimos la visión de Davis (2003:1368) acerca del proceso de sociabilización que debe implicar todo aprendizaje de lenguas. Es decir, no se trata solo de conocer las reglas de uso, sino que es esencial entender las diversas funciones del lenguaje, con tal de poder establecer relaciones, mostrar la pertenencia a un grupo y reforzar la propia identidad. En este sentido, el humor sería un fenómeno pragmático que puede favorecer dicha sociabilización durante la adquisición de una segunda lengua, ya que aparece de forma recurrente en la interacción diaria. De acuerdo con Askildson (2005: 43), la introducción del humor en la enseñanza de una segunda lengua disminuye el filtro afectivo y estimula las actitudes prosociales, esenciales para el buen desarrollo de la comunicación en la L2.

No obstante, a nuestro modo de ver, ser competente a nivel metapragmático y humorístico en la conversación cotidiana sería el último peldaño del proceso. Previamente, los hablantes no nativos deben adquirir determinados conocimientos lingüísticos y socioculturales, así como estar familiarizados con otras manifestaciones humorísticas más sencillas y breves, como puedan ser los chistes o las viñetas cómicas. Resulta, pues, sorprendente que la mayoría de estudios sobre la producción del humor por parte de hablantes no nativos se hayan centrado en estudios de caso con datos extraídos de conversaciones coloquiales (cfr. Davis, 2003; Shardakova, 2010; Shilvery, 2013, Reddington y Waring, 2015), y no se haya estudiado la producción oral ni escrita del chiste en L2. Igualmente, en el campo de la 
apreciación de humor, los trabajos realizados por Bell y Attardo (2010), Gironzetti (2013) y Carbajal (2013) no profundizan demasiado en cómo las variables género, edad, lengua materna e inmersión inciden en este fenómeno cognitivo.

En este sentido, nuestro trabajo se presenta como un estudio innovador dentro de la lingüística aplicada, así como de los estudios sobre la metapragmática del humor, ya que ahonda en un aspecto escasamente tratado en el ámbito del aprendizaje de lenguas extranjeras. El principal propósito de la presente investigación será analizar la competencia humorística de 71 adolescentes, estudiantes de E/L2, a través de los parámetros de la apreciación y producción del chiste. Se trata de un objetivo de carácter bidireccional, puesto que, por un lado, nuestra investigación aúna estas dos fases cognitivas del humor para examinar los principales factores que inciden en la evolución de la competencia humorística, dentro de un contexto de inmersión lingüística, y, por otro lado, intenta aportar algo de luz sobre la función del humor verbal en la activación de las habilidades metapragmáticas en L2.

Para tal fin, en primer lugar, realizaremos una revisión de los principales estudios sobre la metapragmática del humor, para a continuación reflexionar sobre cómo influye esta en la apreciación y producción del humor en la L2, y proponer un conjunto de hipótesis (§2). Sobre estos presupuestos, en el siguiente apartado (§3) expondremos el diseño de nuestra investigación y las técnicas metodológicas empleadas durante el proceso de recogida de datos. Asimismo, presentaremos los principales rasgos del alumnado y de los centros educativos que participaron en la investigación. Después de haber expuesto nuestra metodología, se analizarán en profundidad los resultados obtenidos con el propósito de constatar si nuestras hipótesis eran ciertas o no $(\S 4)$. Unas reflexiones finales sobre la importancia de estimular las habilidades metapragmáticas del humor en el aula de ELE ocuparán las últimas páginas a modo de conclusión (\$5).

\section{LA COMPETENCIA METAPRAGMÁTICA DEL HUMOR EN L2}

Este trabajo parte de la concepción del humor como un fenómeno pragmático basado en una incongruencia, lo cual significa que el humor surge a raíz de una realidad que se presenta como inesperada e incongruente para el lector (cfr. Raskin, 1985; Attardo, 1994; Ruiz Gurillo, 2012). El efecto humorístico se consigue, pues, una vez que oyente o lector activa una serie de inferencias para lograr resolverla, lo cual sería imposible si no estuviésemos dotados de conciencia metapragmática. Este concepto fue introducido por Verschueren (2000) para aludir a la capacidad inherente del ser humano para autorregular su propio uso del sistema lingüístico con fines comunicativos, y para reflexionar sobre las funciones del lenguaje. La conciencia metapragmática se va desarrollando progresivamente durante el periodo de maduración cognitiva y nos permite ser conscientes de qué elementos lingüísticos y extralingüísticos debemos emplear, en un contexto concreto, para que nuestro mensaje consiga en el interlocutor el efecto perlocutivo deseado (cfr. Verschueren, 2000; Crespo et alii. 2010). No obstante, se trata de una noción bastante abstracta debido a la incapacidad para medir dicha conciencia (Verschueren, 2000). En este marco, no cabe duda de que uno de los procesos reflexivos que lleva a cabo el ser humano es el humor, pues en él se activan las habilidades metapragmáticas. Ruiz Gurillo (2016a) nos presenta el humor verbal como un procedimiento de carácter metapragmático que implica una reflexión activa sobre los recursos de conocimiento que emplea el hablante o escritor e interpreta el oyente o lector. Así 
pues, el efecto humorístico es consecuencia directa de las continuas elecciones lingüísticas y extralingüísticas que realiza el escritor o hablante en su producción humorística, y que interpreta y aprecia el oyente o lector. Si atendemos a la nomenclatura desarrollada por el grupo de investigación GRIALE, las elecciones lingüísticas (léxicas, morfosintácticas y fónicas) que realiza el hablante o escritor del mensaje serían indicadores de humor, entendidos estos como procedimientos de por sí humorísticos (cfr. Ruiz Gurillo, 2010, 2012, 2014). Asimismo, en la revisión de la Teoría General del Humor Verbal (TGHV), Ruiz Gurillo (2012) alude a elementos paralingüísticos, kinésicos y tipográficos como marcas que acompañan al humor y colaboran en su inferencia.

Siguiendo las ideas de Gombert (1992), la conciencia metapragmática de los hablantes nativos empieza a construirse en la infancia y va madurando a lo largo de cuatro fases graduales. En particular, el ser humano transcurre progresivamente por la adquisición inicial, la comunicación epipragmática, la competencia metapragmática y la automatización de la competencia metapragmática. Si bien en la fase epipragmática, la persona no discierne completamente el lenguaje literal del figurado y no es todavía capaz de aportar un razonamiento pragmático de sus elecciones lingüísticas (cfr. McGhee, 2002; Timofeeva, 2014). Es en la siguiente fase, la metapragmática, cuando el usuario proporciona explicaciones explícitas sobre el uso de su sistema lingüístico tanto desde el punto de vista productivo como explicativo (cfr. Orekoya et alii.,2014: 65). En esta línea, estudios como el realizado por el grupo de investigación GRIALE ${ }^{1}$ avalan que las niñas y niños de 8 a 12 años ya muestran una competencia metapragmática bastante elaborada, siendo capaces de elaborar textos humorísticos en los que consiguen generar una incongruencia con el empleo de determinados indicadores y marcas (cfr. Timofeeva, 2016, 2017; Ruiz Gurillo, 2015). No obstante, el conocimiento consciente sobre el funcionamiento del lenguaje no es el mismo en todos los usuarios. Así pues, nos formulamos la siguiente pregunta: ¿de qué factores dependerá el desarrollo de las habilidades metapragmáticas en el caso de los hablantes no nativos? Con tal de dar respuesta a este interrogante, examinaremos el papel que juegan factores como el nivel de lengua, la inmersión lingüística, la edad, el género o las competencias intercultural y sociopragmática.

\subsection{La apreciación del humor en L2}

Otro de los pilares teóricos sobre los que se apoya nuestro trabajo sería la propuesta de Hay (2000) sobre las fases cognitivas por las que trascurre, de forma gradual, el interlocutor al recibir un enunciado humorístico. Según la autora, el receptor del mensaje humorístico transcurre progresivamente por las fases de reconocimiento, entendimiento, apreciación y acuerdo. A su vez, Attardo (2001: 166) inserta los procesos cognitivos de reconocimiento, entendimiento, apreciación y acuerdo dentro de la competencia humorística (humor competence) y actuación humorística (humor performance). A su entender, los fenómenos cognitivos de reconocimiento y entendimiento del humor forman parte de la competencia humorística, mientras que la apreciación sería un fenómeno híbrido entre la competencia

1 Grupo de Investigación sobre la ironía y el humor en español del Departamento de Filología Española, Lingüística General y Teoría de la Literatura de la Universidad de Alicante. Para más datos sobre el grupo de investigación GRIALE, puede consultarse su web: http://dfelg.ua.es/griale/. 
humorística y la actuación del humor, puesto que de esta deriva bien el acuerdo ${ }^{2}$ con lo enunciado por el hablante, o bien humor fallido si no considera divertido o gracioso lo dicho. Por su parte, la producción del humor se relacionaría con las fases de reconocimiento, comprensión y apreciación cuando se trata de humor conversacional, en el que el oyente, tras manifestar su acuerdo con lo enunciado, efectúa una réplica en modo humorístico. No obstante, este último fenómeno se puede estudiar de forma más particularizada, desde la perspectiva del hablante o escritor, a través de discursos humorísticos planificados como el chiste, el monólogo o la parodia.

\section{ACTUACIÓN DEL HUMOR}

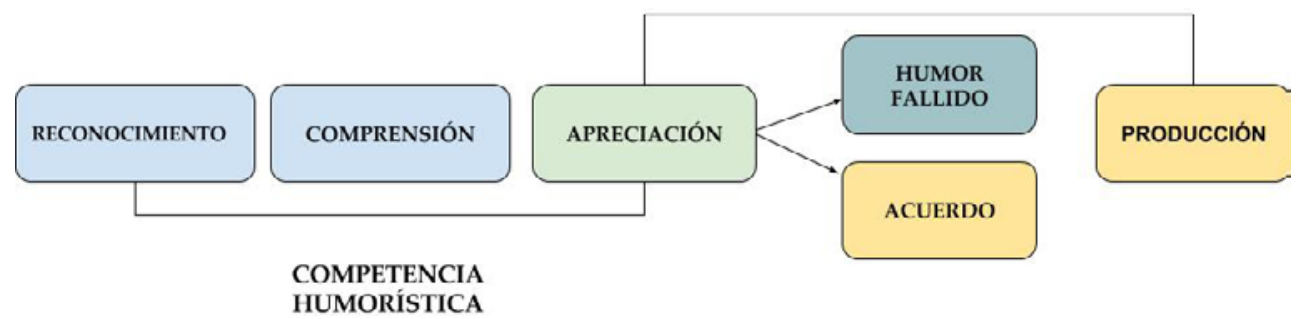

Figura 1. Procesos cognitivos del humor.

Así pues, siguiendo el esquema, nuestro alumnado debe, en primer lugar, reconocer el texto como humorístico, para a continuación poder comprender el contenido no literal del mensaje y, finalmente, decidir si considera que dicha expresión es graciosa o no. En esta última fase, es necesario ser conscientes de que el humor no siempre consigue su propósito de entretener y hacernos reír. Bell y Attardo (2010); Bell (2013) y Carbajal (2013) han identificado las principales razones por las que se produce el humor fallido, esto es, situaciones en las que el interlocutor extranjero no consigue activar las inferencias necesarias para lograr resolver la incongruencia y desencadenar el efecto humorístico deseado. Según estos autores, a veces reconocemos el humor, pero no nos resulta divertido porque no hemos ido más allá del lenguaje literal, o porque no tenemos el suficiente conocimiento cultural o apego emocional por el tema sobre el que gira el chiste. En definitiva, si el alumnado no cuenta con un amplio repertorio léxico-semántico y una adecuada competencia pragmática, resultará imposible la resolución de la incongruencia y, por ende, la apreciación del humor. Asimismo, la capacidad de producir muestras humorísticas como los chistes requiere no solo tener dichos conocimientos, sino la capacidad de ponerlos en práctica para conseguir el efecto humorístico esperado.

\subsection{La producción del humor en L2}

La apreciación y producción del humor en una L2 son tareas de gran complejidad, incluso para aquellos estudiantes de nivel avanzado en la L2 (cfr. Bell, 2005; Shardakova, 2010). Resulta complicado producir secuencias humorísticas en conversaciones coloquiales, dentro de

2 Las expresiones más comunes para expresar dicho acuerdo serían la sonrisa, la risa, o incluso los aplausos si se trata de un mensaje humorístico emitido ante un público. 
un contexto informal en E/L2, si no tenemos el bagaje cultural necesario y la adecuada competencia metapragmática, esto es, la capacidad para reflexionar sobre el uso y las funciones del sistema lingüístico empleado por el emisor para generar el efecto humorístico. En esta línea, Davis (2003:1361) habla del estado de vulnerabilidad del hablante extranjero en un contexto de conversación humorística en L2 con hablantes nativos. Por ello, consideramos esencial que los aprendices adquieran, durante sus clases de ELE, las herramientas lingüísticas básicas para poder identificar las marcas e indicadores característicos del humor verbal. De esta forma, no solo serán capaces de interpretar las inferencias y resolver las incongruencias del texto humorístico, sino que podrán intentar producir sus propias muestras humorísticas. De lo contrario, es decir, si no están familiarizados con los usos y funciones del lenguaje humorístico, además de ser incapaces de producir humor en la L2, corremos el riesgo de que tomen el mensaje de su emisor de forma literal y se frustren al no encontrarle el sentido.Por todo ello, en esta investigación examinaremos qué factores intervienen en el uso de los diferentes indicadores y marcas humorísticos a la hora de contar un chiste en E/L2 (§4.2).

En resumen, el presente trabajo parte de las fases del humor que presenta Hay (2000) y la clasificación de estas, propuesta por Attardo (2001). Además, otra de las bases teóricas sobre los que se sostiene esta investigación es el modelo de la metapragmática del humor planteado por Ruiz Gurillo (2016a: 90-91), según el cual el oyente o lector reflexiona, de forma consciente o no, sobre el uso lingüístico que hace el hablante o escritor en una determinada situación humorística, lo que lo convierte en competente a nivel metapragmático cuando logra inferir y apreciar el humor. Asimismo, el hablante o escritor realiza un ejercicio reflexivo sobre el lenguaje que emplea para alcanzar el efecto perlocutivo deseado en el interlocutor. Así pues, consideramos que las redes cognitivas que activan los usuarios no nativos a la hora de inferir, apreciar y producir humor dentro de un contexto requieren de una gran capacidad metapragmática.

Estos pilares teóricos nos llevan a plantearnos tres hipótesis de partida. En primer lugar, nuestra investigación cuenta con la hipótesis general de que variables como nivel de lengua, el grado de inmersión lingüística, la lengua materna de los estudiantes, el género, el tiempo que lleve el aprendiz en nuestro país, o el curso académico condicionan e inciden directamente en los procesos cognitivos de apreciación y producción del humor verbal. En esta línea, sostenemos como segunda hipótesis, la idea de que encontraremos evidencias que denoten rasgos epipragmáticos y metapragmáticos no tanto en base de la edad de estos, sino en relación con su nivel de lengua y grado de inmersión lingüística. En este caso, la competencia metapragmática de los aprendientes de E/L2, que permite descifrar el mensaje humorístico, estará condicionada por los conocimientos adquiridos previamente en relación con la L2 y de acuerdo con la capacidad de los usuarios para transferir sus conocimientos y habilidades de la L1 a la L2. Por último, defendemos la hipótesis de que la producción del humor en E/L2 implica un salto en las habilidades metapragmáticas y cognitivas, puesto que el hablante debe emplear el lenguaje específico para una determinada situación en su propósito de divertir o hacer reír al receptor del mensaje humorístico. En este caso, no solo se trata de reconocer los mecanismos lingüísticos y paralingüísticos empleados, sino que el alumnado de E/L2 que haga uso del humor deberá tener un dominio adecuado de los diversos recursos fónicos, léxicos y sintácticos que conforman los indicadores del humor, así como usar determinados elementos extralingüísticos y tipográficos para situar al lector en modo humorístico. 


\section{EL CHISTE COMO HERRAMIENTA PARA EL DESARROLLO DE LA COM- PETENCIA HUMORÍSTICA}

Para Brown y Levinson (1987), el chiste tiene una conexión directa con la cortesía positiva, pues favorece la afiliación grupal y el sentimiento de camaradería, cuando ambas partes se divierten con la broma. Sin embargo, la incapacidad de captar la incongruencia sobre la que gira el chiste suele producir el efecto contrario y generar cierta incomodidad y malestar en el interlocutor, quien puede incluso sentir que se le está evaluando su competencia humorística (cfr. Sacks, 1974; Zajdman, 1995). Resulta, así, evidente la necesidad de acercar a los estudiantes al humor desde el aula de E/L2. Diversos estudios avalan sus beneficios a nivel cognitivo y psicológico, ya que se ha comprobado que favorece la cohesión de grupo (cfr. Jefferson, 1984; Schmitz, 2002), reduce la ansiedad y el estrés al que se pueden ver sometidos en determinadas situaciones durante el proceso de aprendizaje (cfr. Deneire, 1995; Lefcourt, 2001) y promueve el desarrollo de la inteligencia emocional, habilidad muy ponderada en nuestro actual sistema educativo. De hecho, de acuerdo con Yip y Martin (2006), la gente con mejor sentido del humor demuestra una mayor inteligencia emocional y es más competente a nivel interpersonal.

Asimismo, la decisión de centrar nuestra investigación en el género humorístico del chiste vino motivada por su naturaleza breve, precisa y sencilla. En concreto, el chiste que seleccionamos para analizar la apreciación del humor en E/L2 es un enunciado que juega con la ambigüedad que producen las palabras homónimas cobra (tercera persona del singular del verbo cobrar) y cobra (tipo de serpiente). La comprensión de este chiste no requiere de ningún conocimiento sociocultural específico, por lo que los estudiantes solo necesitaron acudir a sus recursos léxico-semánticos para llegar a captar la gracia. Nuestro ejemplo ${ }^{3}$ estaba contado por el cómico Leo Harlem durante el programa Zapeando emitido por La Sexta. En este caso, creímos conveniente presentar nuestro ejemplo en formato audiovisual con el fin de que los aprendientes se sirvieran de las marcas paralingüísticas y kinésicas empleadas por el humorista a la hora de captar el sentido y la intención del mensaje. Es evidente que la correcta comprensión auditiva del mensaje supone el papel activo del oyente, quien debe interpretar el enunciado teniendo en cuenta determinados aspectos extralingüísticos propios del discurso oral (cfr. Martin Peris, 1991).

\subsection{Principales rasgos de los participantes objeto de estudio}

Los resultados que a continuación presentamos han sido recogidos en ocho centros educativos plurilingües ubicados en distintos puntos de la Comunidad Valenciana y de la Región de Murcia. En total, hemos contado con la participación de 71 estudiantes de E/L2 que se encontraban cursando Bachillerato o Educación Secundaria Obligatoria (en adelante, ESO) durante el curso 2016/2017. Así pues, los informantes del estudio son adolescentes con edades comprendidas entre los 12 y los 18 años, que suman una media de 14'8 años, entre todos ellos. Consideramos que la franja de edad que abarcan estos

3 Durante la criba del material, tuvimos en cuenta factores que pudieran dificultar en exceso la comprensión. Entre los elementos que nos ayudaron a descartar determinadas muestras se encuentran: la dicción de los humoristas y su velocidad del habla, los juegos de palabras empleados y las referencias a elementos culturales desconocidos para los alumnos internacionales. 
cursos escolares era la más ventajosa para nuestro estudio, ya que, durante este período, los adolescentes -como ellos mismos reconocieron- tienen un contacto continuo con los géneros humorísticos a través de los chistes y memes en las redes sociales, las sitcoms y los vídeos de YouTube. Además, nuestra decisión de trabajar con este grupo de edad vino motivada por las ideas presentadas en los trabajos de Orekoya et al. (2014: 65) y Hess (2016: 6). De acuerdo con los investigadores, es en la adolescencia cuando nuestro pensamiento alcanza mayor abstracción y se rige por principios lógicos, lo que implica que nuestros participantes han adquirido ya la capacidad para detectar las incongruencias en el discurso y divertirse con chistes más complejos a nivel cognitivo y lingüístico (cfr. Martin, 2007: 241).

No obstante, de acuerdo con nuestra primera hipótesis, la competencia humorística no solo se verá afectada por el factor edad, sino también por otras variables como la nacionalidad, el sexo, el nivel de lengua o el grado de inmersión lingüística. En este sentido, la procedencia de los estudiantes podrá favorecer, en algunos casos, la apreciación y producción del humor verbal español. De hecho, para aquellas personas que hablan una lengua romance, como el francés, el portugués o el italiano, puede que les resulte más sencillo entender el contenido del mensaje. En nuestro caso, los participantes procedían de todos los rincones del mundo. El grupo más numeroso lo conformaban un total de 32 estudiantes procedentes de países anglófonos, seguidos por 14 rusófonos, dies francófonos, 6 sinohablantes, 3 lusófonos, 2 germanohablantes, 2 neerlandófonos y otros 2 italianófolos. Asimismo, debemos señalar que se descartaron dos de las respuestas del estudio, ya que uno de los estudiantes procedía de un país de habla hispana y el otro había nacido en España, pese a tener raíces asiáticas.

Otro factor que hemos tenido en consideración en esta investigación es el género del alumnado. Pese a haber más similitudes que diferencias entre ambos sexos en cuanto a la competencia y la actuación del humor (Crawford, 2003), conviene analizar en qué medida difiere la apreciación humorística de nuestras alumnas y alumnos atendiendo a esta variable. En este caso, el análisis de nuestros datos en relación con la variable género se ha visto favorecida por un número equiparado de participantes en ambos géneros, ya que el 50'7\% de los informantes eran varones y el $49^{\prime} 3 \%$ fueron mujeres.

Del mismo modo, el grado de inmersión lingüística en el que se encuentran nuestros estudiantes será un elemento condicionante. De acuerdo con el Diccionario de Términos Clave $^{4}$ del Centro Virtual Cervantes, "se habla de inmersión total cuando la L2 es la lengua vehicular dominante en el currículo, y de inmersión parcial, cuando la primera y segunda lengua funcionan de manera alternativa como medio de comunicación en el aula". No cabe duda de que una situación en inmersión lingüística plena favorecerá el proceso madurativo de las habilidades metapragmáticas y la comprensión del humor en L2. En este sentido, Pastor Cesteros (2004) afirma que el uso de la lengua meta como vehículo de enseñanza influye en la adquisición de la competencia sociolingüística, en el desarrollo de la competencia comunicativa y, por tanto, en el aprendizaje de la L2.

4 Enlace de acceso a la definición: < http://cvc.cervantes.es/ensenanza/biblioteca_ele/diccio_ele/diccionario/ inmersionlinguistica.htm> 


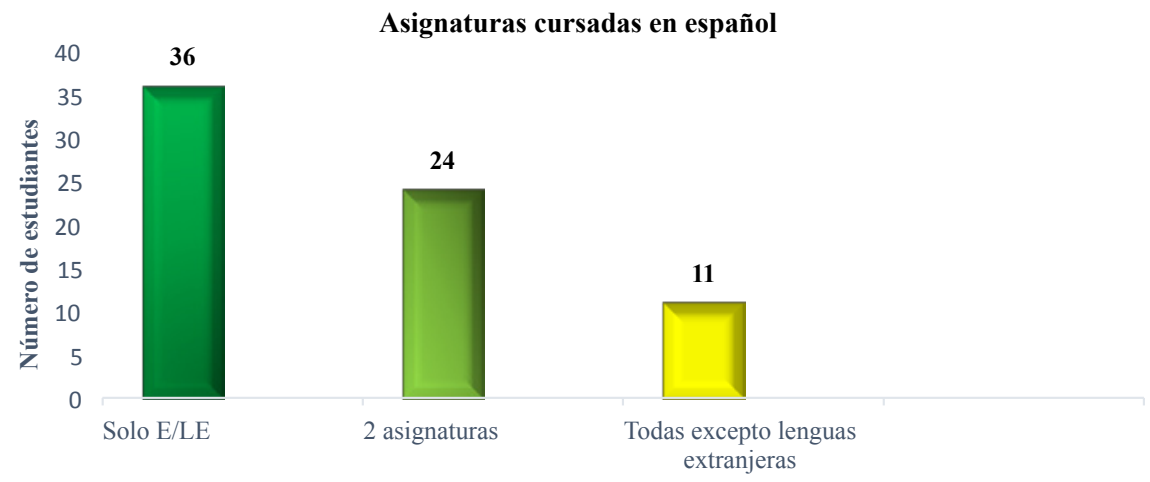

Gráfico 1. Número de estudiantes en relación con las asignaturas que cursan en español.

Así pues, observando el gráfico 1, podemos concluir que la gran mayoría de los encuestados no se encuentran en plena inmersión lingüística, ya que tan solo 11 de ellos cursan el grueso de sus asignaturas en español. No obstante, debemos reconocer que la oportunidad de estar en contacto directo con la lengua fuera del aula favorece en gran medida el proceso el aprendizaje. De ahí que el nivel de los estudiantes se vea condicionado tanto por el grado de inmersión como por su relación con el entorno social fuera del aula ${ }^{5}$.

En cuanto al nivel de lengua ${ }^{6}$, los datos recogidos cifran en una mayoría simple a los estudiantes con nivel umbral B1 (35'2\%), seguidos por el alumnado con nivel B2 (28'2\%) y aquellos con nivel elemental A2 (25,4\%). Los porcentajes descienden en los niveles superiores, con tan solo un $10 \%$ de estudiantes con nivel avanzado $\mathrm{C} 1$ y un 1 ' $2 \%$ con nivel maestría $\mathrm{C}^{7}$. De acuerdo con estos datos, comprobaremos en el siguiente apartado si la competencia humorística en español como L2 puede adquirirse desde niveles elementales, lo cual rebatiría lo expuesto en el Plan Curricular del Instituto Cervantes (en adelante, PCIC) y el Marco Común Europeo de Referencia (en adelante, $M C E R^{8}$ ) y reforzaría las ideas de Davis (2003), Schmitz (2002), Bell (2009) y del grupo de investigación GRIALE (2011), quienes están convencidos de que el contacto con pequeñas muestras irónico-humorísticas, como los chistes breves o las viñetas cómicas, debe iniciarse desde los niveles más inferiores.

5 Aunque la lengua meta está en el entorno inmediato del aprendiz y está expuesto a ella continuamente, varios de los docentes con los que tuve la oportunidad de compartir impresiones, manifestaron su inquietud por la situación de aislamiento a modo de burbuja en la que muchos de los estudiantes vivían dentro de sus comunidades.

6 Los datos que recogimos sobre nivel de lengua fueron corroborados por el profesorado. En muchos casos, los estudiantes no tenían su nivel acreditado con ninguna certificación; sin embargo, gracias al manual de texto, pudo determinarse en qué escalón del Marco Común Europeo de Referencia se situaban.

7 El único caso de nivel maestría C2 se dio en una adolescente italiana que llevaba viviendo en España de cinco a seis años y cursaba $2^{\circ}$ de Bachillerato en un centro educativo con inmersión lingüística plena.

8 Ambos documentos son obras de referencia para cualquier docente de ELE, sin embargo, el humor no recibe la atención necesaria en ninguna de estas guías y su enseñanza se sitúa en los niveles intermedio alto (B2) y avanzado (C1) (cfr. Ruiz Gurillo, 2008). El MCER dedica un apartado a las competencias pragmáticas de la lengua e inserta el uso del humor como un componente a valorar en la adecuación sociolingüística del nivel avanzado C1 (MCER, 2002:119). Por el otro lado, en el apartado de Géneros discursivos del PCIC, se nos insta a enseñar aspectos referidos al humor en el aula a partir del nivel B2, acotando dicha enseñanza a chistes sin implicaciones socioculturales, Para mayor detalle, consúltese http://cvc.cervantes.es/ensenanza/biblioteca_ele/plan_curricular/ niveles/07_generos_discursivos_inventario_b1-b2.htm $($ PCIC, 2006). 


\section{Nivel de español L2/LE}

$40,0 \%$
$35,0 \%$
$30,0 \%$
$25,0 \%$
$20,0 \%$
$15,0 \%$
$10,0 \%$
$5,0 \%$
$0,0 \%$
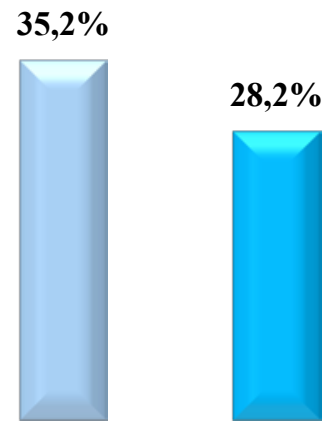

Nivel A2

Nivel B1

Nivel B2

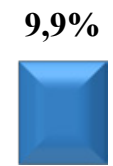

Nivel C1
$1,4 \%$

Nivel C2

Gráfico 2. Nivel de español de los estudiantes.

\subsection{Diseño de la investigación}

Como hemos visto en el epígrafe 2.1, la apreciación del humor se sitúa entre la competencia del humor y la actuación del humor. Se trata del paso que puede desencadenar, bien en un efecto hilarante y en una posible respuesta por parte del interlocutor, o bien en humor fallido. Por consiguiente, la observación de este fenómeno ha sido uno de los elementos clave en nuestro estudio. La metodología empleada para llevar a cabo dicha investigación es de carácter hipotético-deductiva, lo que conlleva que sea, a su vez, exploratoria, descriptiva y contrastiva. Diseñamos una plantilla de observación ${ }^{9}$ con el propósito de analizar cómo se manifestaba el fenómeno de apreciación del humor en los escolares no nativos. Al utilizarla durante el primer visionado, los datos obtenidos corresponden a sus primeras reacciones ante el enunciado humorístico - previas a que se vieran influidos por los comentarios y explicaciones del resto de compañeros. En el primer apartado, registramos información sobre el tipo de centro educativo, el curso escolar, el nivel de español y el sexo del estudiante. Seguidamente, prestamos también atención a factores internos del aprendizaje ${ }^{10}$ relacionados con la motivación y disposición del alumnado hacia la actividad, puesto que ello, sin duda, influye a la hora de situarse en modo humorístico y apreciar el humor. Finalmente, atendimos a la expresión de sonrisas, risas, gestos faciales y comentarios del tipo "no lo entiendo", como señales de apreciación del humor.

9 Somos conscientes de que existen otros instrumentos para la medición de la apreciación del humor que han sido validados. De hecho, las primeras escalas y tests para medir este fenómeno en chistes y viñetas cómicas se crearon a mediados del siglo XX (cfr. Andrews, 1943; Eysenck, 1942). Asimismo, en la actualidad, han adquirido gran reconocimiento la Escala de Apreciación del Humor (EAHU) elaborada por Dios Carretero (2005) y el Humor Styles Questionnaire (HSQ) diseñado por Martin, et al. (2003). No obstante, todos estos instrumentos fueron elaborados desde una perspectiva puramente psicológica y están pensados para emplearse con hablantes nativos.

10 El Diccionario de términos clave de ELE (Martín Peris 2008) señala como factores internos del aprendizaje: la motivación del aprendiente, sus necesidades y su estilo de aprendizaje. Mientras que determina como factores externos el contexto en el que el aprendizaje se produce, así como la enseñanza que intenta promoverlo. 
La sonrisa es un factor que apenas había sido tenido en cuenta hasta hace poco. En los últimos años, la sonrisa se ha convertido en objeto de numerosos estudios como, por ejemplo, los trabajos realizados con la técnica de eye-tracking (cfr. Gironzetti, et.al., 2016). Sin embargo, se trata de una pista que no deja de llevar adherida cierta problemática, relacionada con la precisión y objetividad científica. Como comentábamos al inicio, tener buen sentido del humor es un rasgo de la personalidad del ser humano que ha estado siempre altamente ponderado; por consiguiente, podemos en ocasiones tender a fingir apreciación, bien por salvaguardar nuestra imagen, o bien por estrechar lazos con el interlocutor si se trata de una conversación (cfr. Sacks, 1974; Hay, 2001; Bell, 2013). A pesar de esto, con todas las prevenciones, decidimos tomar la acción no verbal de sonreír como indicio de aprecio y respaldo al contenido humorístico expuesto.

De igual forma, la risa es, sin duda, un referente en la apreciación del humor, ya que es una de las respuestas más comunes ante algo que nos parece ocurrente o gracioso. De hecho, nadie duda de que la risa es el indicio contextual de apreciación del humor por excelencia y autores como Bergson, et al. (1911) ya hablaban de la risa como un fenómeno social que refleja un apreciamiento del humor. Además, compartimos la idea de Hay (2001:76) cuando dice que "laughter is very tightly associated with appreciation. You can laugh, and then deny agreement, but you can't laugh, and then deny appreciation." No obstante, este elemento también entraña cierta controversia, puesto que no siempre que nos reímos significa que compartimos lo que dice el texto. A veces, la risa puede ser señal de sorpresa, vergüenza o distancia ante lo enunciado (cfr. Attardo, 2003; Coates, 2007), o incluso puede ser un indicio sesgado para salvaguardar nuestra imagen frente al hablante. Estas ideas quedan perfectamente resumidas en la siguiente cita de Kotthoff (2006:4):

When I tell someone a joke, I watch for signs of amusement. If the listener is willing to cooperate, s/he will let me finish, and then smile, laugh, or respond in some way to the humorous potential. If the listener did otherwise, she would define my joke as a dud. It is this dialogicity, which inhibits timid people, strangers, or people of lower status from joking in many contexts. They fear the embarrassment of not eliciting the appropriate reaction.

En cuanto a las marcas de humor fallido, consideramos oportuno fijar nuestra atención en las expresiones faciales y comentarios, con el objetivo de advertir cualquier señal de incomprensión o rechazo ante lo dicho en los vídeos. Aunque somos conscientes de que el silencio puede ser también una marca de humor fallido (cfr. Kotthoff, 2006; Bell, 2010), preferimos no tenerlo en cuenta porque, en esta ocasión, los participantes únicamente recibían input y no se les pidió que respondiesen ante lo dicho, es decir, la dialogicidad no era obligatoria.

No obstante, y como es lógico, el estudio de este proceso cognitivo, a través de este instrumento, se vio limitado por nuestra capacidad de observación. De modo que, pese a tener a un total 71 estudiantes con los que poder realizar esta prueba, tuvimos que reducir el número de informantes a un máximo de 5 por clase con el fin de intentar atender al mayor número de señales y expresiones de apreciación. La elección de estos se realizó de forma aleatoria, intentando siempre buscar la paridad en el género. En total, contamos con la posibilidad de 
estudiar al detalle el comportamiento de 22 estudiantes, 13 de sexo masculino y 9 de sexo femenino. La siguiente tabla resume los rasgos principales de dichos informantes:

\begin{tabular}{|c|c|}
\hline COLEGIO & $\begin{array}{c}\mathbf{N}^{{ }^{\boldsymbol{}}} \\
\text { alumnos }\end{array}$ \\
\hline Xabia International College & 2 \\
\hline El Limonar International School & 5 \\
\hline American School of Valencia & 4 \\
\hline Laude Newton College & 2 \\
\hline Caxton College & 2 \\
\hline Liceo Francés de Alicante & 2 \\
\hline King's College of Murcia & 3 \\
\hline Colegio Internacional los Naranjos & 2 \\
\hline TOTAL & 22 \\
\hline
\end{tabular}

\begin{tabular}{|c|c|}
\hline EDAD & $\begin{array}{c}\mathbf{N}^{\mathbf{o}} \\
\text { alumnos }\end{array}$ \\
\hline 14 años & 3 \\
\hline 15 años & 11 \\
\hline 16 años & 1 \\
\hline 17 años & 3 \\
\hline 18 años & 4 \\
\hline TOTAL & $\mathbf{2 2}$ \\
\hline
\end{tabular}

\begin{tabular}{|c|c|} 
NIVEL & $\begin{array}{c}\mathbf{N}^{\mathbf{0}} \\
\text { alumnos }\end{array}$ \\
\hline $\mathrm{A} 2$ & 3 \\
\hline $\mathrm{B} 1$ & 9 \\
\hline $\mathrm{B} 2$ & 5 \\
\hline $\mathrm{C} 1$ & 5 \\
\hline TOTAL & 22 \\
\hline
\end{tabular}

Tabla 1. Informantes observados para la apreciación del humor.

Afortunadamente, la recogida de datos se amplió gracias al diseño de un cuestionario online realizado a través de la plataforma digital Survio ${ }^{11}$, el cual ha aportado luz a esta investigación sobre el paradigma de la producción de humor por parte de hablantes de E/L2. El carácter virtual del cuestionario posibilitó que las y los encuestados dispusieran de él en sus dispositivos electrónicos, así como en formato papel si no tenían acceso a la red. El ítem en el que invitábamos a contar un chiste en español-de manera escrita-obtuvo un índice bajo de respuesta y tan solo 17 encuestados se animaron a realizar esta tarea de producción humorística.

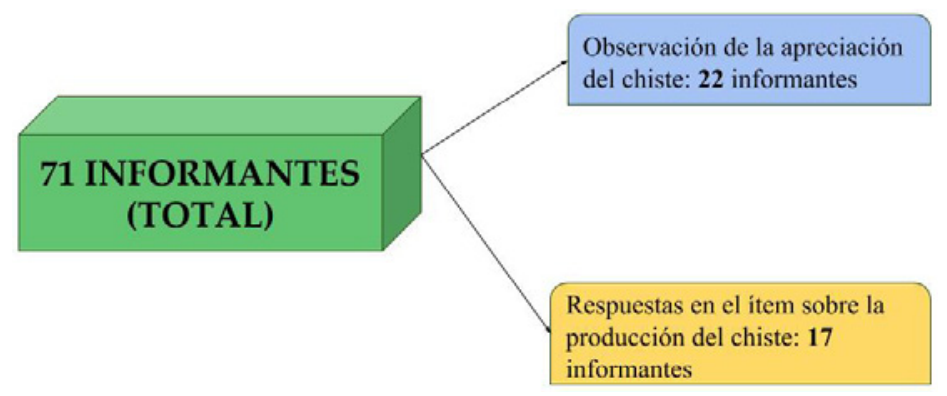

Figura 2. Número de informantes que participaron en el estudio.

En este sentido, el conjunto de métodos nos ha permitido estudiar cómo actúa la conciencia metapragmática durante los procesos de apreciación y producción del humor, así como analizar los recursos lingüísticos y extralingüísticos que los estudiantes son capaces de utilizar dependiendo de factores como su nivel de lengua o el grado de inmersión lingüística, entre otros.

11 Se puede acceder a nuestro cuestionario a través del siguiente enlace: $<\mathrm{https}: / /$ www.survio.com/survey/d/ P4J2O2V4Y9H8S4R3H> 


\section{RESULTADOS Y DISCUSIÓN}

\subsection{La apreciación del humor en E/L2}

En el caso de este chiste, la situación comunicativa ayudó a los oyentes a que se posicionaran en modo humorístico desde el principio. El contexto televisivo, las risas iniciales del público, el uso de diversas marcas kinésicas y la entonación de Leo Harlem fueron las pistas metapragmáticas claves que predispusieron a nuestros informantes para la recepción del chiste. Asimismo, las estrategias narrativas seleccionadas por el humorista contribuyeron en el éxito del mismo. Aunque, sin duda, la clave para inferir el humor estuvo en la elección de los indicadores y marcas que acompañan tanto al gancho como al remate. El humorista recurre a diversos indicadores y marcas de humor para realizar una serie de enunciados humorísticos conocidos como ganchos (o jab lines); asimismo, estos elementos también acompañan al broche final de la narración con el que se desata el efecto cómico, esto es, el remate (o punch line) (Ruiz Gurillo, 2016b: 616).

Se trata de un chiste breve y planificado para ser contado en estilo directo como si fuera un diálogo entre una madre y un hijo. Tomando como referencia la clasificación de los chistes de Attardo (1994), nuestra muestra se clasificaría como un chiste verbal basado en la ambigüedad semántica; en este caso, la apreciación depende exclusivamente de identificar como humorístico el indicador de homonimia en la palabra cobra. La transcripción del texto ha sido realizada siguiendo las claves del sistema Val.Es.Co ${ }^{12}$ :

(1) Público: (RISAS)

FASE DE ESTABLECIMIENTO INCONGRUENCIA Leo Harlem: ¡mamá mamá me ha picao una serpiente! FASE DE RESOLUCIÓN

$\Rightarrow$ dice $\rightarrow$ ¿cobra?

$\longrightarrow$ dice $\rightarrow$ no no/ ;lo ha hecho gratis!

Por un lado, la palabra cobra como tipo de serpiente es homónima de la tercera persona del presente indicativo del verbo "cobrar". Esta homonimia es el indicador lingüístico que provoca, en este caso, una infracción del principio de Informatividad, puesto que se produce una ambigüedad semántica. Por el otro, la repetición de la negación "no" con tono irónico en el remate del chiste supone una inversión del principio de Cantidad, que repercute a su vez en el prerrequisito de Cualidad ("diga la verdad"), y sitúa al interlocutor en modo humorístico.

Sin embargo, los chistes enlatados están normalmente asociados por algunas culturas y grupos sociales al humor simple, es decir, poco elaborado o infantil (cfr. Bell 2013: 188). Puede que esta sea la razón por la que únicamente 12 de los 22 estudiantes observados mostraron señales de apreciación positiva al chiste en su rostro. En este sentido, nos parece interesante destacar que estos informantes, que apreciaron el chiste a través de la sonrisa y/o la risa, mostraban una actitud positiva hacia la actividad y se encontraban cursando el segundo ciclo de ESO o bachillerato. Asimismo, 8 de estos 12 aprendientes presentaban un nivel de español avanzado. El resto del alumnado (10) necesitó de unos segundos para resolver la incongruencia e inferir el humor, ya que la brevedad del chiste no permite al alumno contextualizar el mensaje de la misma forma que pueda hacerlo en géneros más extensos

12 Véase "Claves para el sistema de transcripción" del grupo Val.Es.Co en <http://www.uv.es/valesco/sistema.pdf> 
como el monólogo o la parodia (cfr. Carrel, 1997). Hubo incluso 5 jóvenes que manifestaron su incomprensión mediante sus expresiones faciales y comentarios del tipo “¿Qué? no lo entiendo" o "No lo he pillado". En este último caso, todos ellos fueron varones con un nivel umbral (B1) de español.

\section{Apreciación del chiste}

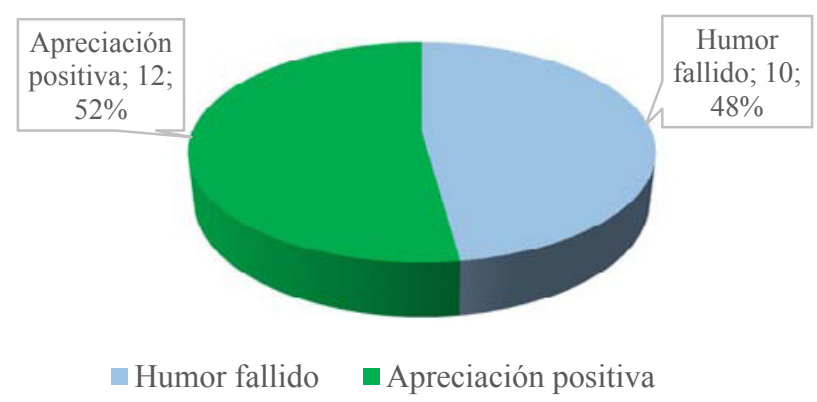

Gráfico 2. Apreciación del chiste.

Si nos basamos en el modelo psicológico de incongruencia-resolución desarrollado por Suls (1972), según el cual para que un hecho sea considerado gracioso, el receptor del mensaje ha de activar unas determinadas inferencias que le ayuden a resolver la incongruencia, no es de extrañar que las mayores muestras de apreciación se dieran en el remate final. De hecho, haciendo una analogía con el boxeo, el remate sería el golpe final que desencadena el efecto hilarante (cfr. Alvarado 2012: 13). Es aquí donde se crea un desajuste o contradicción que lleva al oyente a activar sus habilidades metapragmáticas para poder reinterpretar los ganchos y resolver cognitivamente la incongruencia (Attardo 2001a: 83). En concreto. En definitiva, nuestros resultados demuestran que el chiste es un texto humorístico breve y conciso que se presta para ser introducido en el aula desde los niveles iniciales. Sin duda, el aula es el espacio ideal para experimentar con el humor sin tener la preocupación de confesar que no hemos entendido el mensaje, así, consideramos que el uso del chiste en la clase de español como L2 significaría un primer acercamiento al humor en la lengua meta. Además, este tipo de manifestaciones humorísticas pueden convertirse en un material didáctico ideal para enseñar y ejemplificar determinados fenómenos lingüísticos y culturales propios de la lengua meta. Esto, a su vez, supondrá el desarrollo de la competencia metapragmática del humor, puesto que, para llegar a apreciar el humor, primero deberán reconocer los indicadores y marcas del humor y entender el enunciado como humorístico.

\subsection{La producción del chiste en $\mathrm{E} / \mathrm{L} 2$}

Nos pareció también interesante analizar la capacidad de producción de humor por parte del alumnado de E/L2. Así pues, animamos a nuestros estudiantes a que nos contaran, por escrito, un chiste al final del cuestionario. De esta forma, conseguimos activar no solo sus habilidades metapragmáticas relacionadas con la competencia humorística, sino también aquellas relacionadas con la producción del humor. Como hemos expuesto anteriormente 
(§2.2), la producción humorística implica un alto grado de competencia metapragmática y sociocultural, que vienen a su vez dadas por factores como el nivel de lengua, la edad o el tiempo de exposición a la lengua meta. Por tanto, no es de extrañar que solamente obtuviéramos 17 respuestas positivas para este ejercicio voluntario. En este caso, la recogida de las muestras se ha visto muy influida por el nivel de español ${ }^{13}$, el curso escolar y la nacionalidad de nuestros informantes. Dada la escasez de respuestas, consideramos oportuno analizarlas desde el paradigma cualitativo.

La siguiente tabla agrupa las respuestas recibidas de acuerdo con el nivel de lengua de los informantes:

\begin{tabular}{|l|l|}
\hline NIVEL PLATAFORMA A2 & $\begin{array}{l}\text { 1. ¿Dónde se van los gatos cuando se mueren? Al purgatorio }{ }^{14} \\
\text { 2. -Padres, me voy a Rusia. - ¿En serio? -No, estoy en Rusia }\end{array}$ \\
\hline NIVEL UMBRAL B1 & $\begin{array}{l}\text { 3. No me recuerdo como tiro el boomerang, pero volvió a mí } \\
\text { 4. ¿Cómo se escribe calcetines? -ESO SI QUE ES (SOCKS) } \\
\text { 5. ¿Qué es una vaca con los ojos cerrados? Leche concentrada } \\
\text { 6. La defensa del Manchester United } \\
\text { 7. La defensa del Real Madrid } \\
\text { 8. Viñeta cómica (Figura 3) }\end{array}$ \\
\hline NIVEL AVANZADO B2 & $\begin{array}{l}\text { 9. ¿Cómo Harry Potter baja una colina? Andando. J.K., Rolling } \\
\text { 10. ¿Porque el show se llama Bob Esponja si Patrich (Patricio) es la } \\
\text { estrella? }\end{array}$ \\
& $\begin{array}{l}\text { 11. La profe le dice a Jaimito: - ¿Qué tiempo es "esto no debería } \\
\text { haber pasado"-Preservativo imperfecto }\end{array}$ \\
& $\begin{array}{l}\text { 12. ¿Qué has escrito en el ecsamen? -he dejado mi papel blanca } \\
\text {-No tio, ahora el profe va a decir que hemos copiado! }\end{array}$ \\
& $\begin{array}{l}\text { 13. Iban dos personas y se calló el del medio } \\
\text { 14. Esto era un hombre con los pelos del culo tan, pero tan largos } \\
\text { que un día se tiró un pedo y murió a latigazos. }\end{array}$ \\
\hline NIVEL DOMINIO C1 & $\begin{array}{l}\text { 15. Dos patos se van a casar, uno le dice al otro "nos vamos a casar } \\
\text { pato la vida" }\end{array}$ \\
\hline NIVEL MAESTRÍA C2 & $\begin{array}{l}\text { 16. ¿Cuál es la diferencia entre un hombre y un plátano? Que los } \\
\text { plátanos maduran. } \\
\text { 17. ¿Cómo se suicida un electrón? Tirándose por un puente de } \\
\text { hidrógeno. }\end{array}$ \\
\hline
\end{tabular}

Tabla 2 Chistes escritos por estudiantes de E/L2.

Aunque la tipología de los chistes, por lo general, no difiere demasiado en relación con el nivel de lengua, sí que nos parece interesante destacar las diferencias entre los chistes producidos en el nivel plataforma y en el nivel maestría; así como los rasgos de los informantes, ya que en ambos casos fueron las mujeres quienes los escribieron. En nivel A2, se trató de dos alumnas rusas de 14 años que estudiaban en inmersión lingüística parcial y

132 de los chistes fueron escritos por parte de aprendientes con nivel plataforma A2, 6 por estudiantes en nivel umbral B1, 5 por aprendices en nivel avanzado B2, 2 de nivel dominio C1, y las dos últimas respuestas las dio una alumna con nivel maestría C2.

14 Los chistes aquí plasmados han sido transcritos conforme los escribieron los estudiantes. 
llevaban escasos dos años en España. Ello refuerza la idea defendida por Davis (2003) de que la competencia humorística se adquiere desde niveles iniciales y que es posible promover la competencia metapragmática desde el aula de E/L2. No obstante, es cierto que la temática y la complejidad de los chistes varía de forma considerable en comparación con las muestras de humor producidas por la alumna de nivel C2, quien se atrevió a contar dos chistes: uno de ellos feminista y el otro relacionado con la asignatura de Física y Química. Recordamos que esta informante de 17 años era de nacionalidad italiana, llevaba en España 5 años y cursaba sus estudios en un centro educativo de inmersión lingüística plena, por lo que su maduración metapragmática es ya prácticamente total.

Por otra parte, el pilotaje de esta tarea nos ha permitido apreciar rasgos de comunicación epipragmática y metapragmática en la producción del humor. Con respecto al primer tipo, la traducción directa de puns $^{15}$ al español y el uso de léxico escatológico podría considerarse una actuación epipragmática (Timofeeva 2016: 280). Así, nos encontramos con respuestas como las del número 3, 9 y 10 de la tabla. En estos tres casos se evidencia cómo la comicidad de los puns se pierde al intentar traducirlos del inglés. Por ejemplo, el chiste $\mathrm{n}^{\circ} 9$ no puede siquiera ser entendido como chiste si no traducimos $\left(J^{16}\right.$, Rolling por "es broma, rodando").

No obstante, se puede observar cómo las muestras de competencia metapragmática fueron más numerosas. La mayoría de los chistes eran de tipo acertijo, es decir, se formula una pregunta cuya respuesta se plantea inesperada e incongruente para el oyente y genera cierta comicidad. En particular, este estilo humorístico podría relacionarse con el último estadio del desarrollo del humor que nos propone McGhee (2002): Stage 5: Riddles and Jokes que, según nuestros datos, en hablantes no nativos se iniciaría desde el nivel A2 y se desarrollaría principalmente a partir del nivel umbral B1. Muestras como la $\mathrm{n}^{\circ} 5$ o la $\mathrm{n}^{\circ} 11$ ejemplifican este estilo de pregunta-respuesta.

Además, en muchos casos, los chistes están basados en distintos juegos de palabras. Se aprecian indicadores que infringen el principio del informatividad como la homonimia, la polisemia, la paronimia o la metáfora, confirmándose, así, el desarrollo de sus habilidades metapragmáticas. En este sentido, algunos de los docentes comentaban que muchos de los chistes los habían aprendido gracias al contacto con el resto de alumnos españoles durante los recreos, lo que demuestra la importancia de la inmersión lingüística en la adquisición de la competencia metapragmática del humor.

Si bien las muestras de nivel más alto de competencia metapragmática se dieron en los casos en los que los alumnos decidieron inventar sus propios chistes. Por un lado, dos alumnos anglófonos emplearon el humor como metalengua para calificar como "chiste" la defensa del Real Madrid y del Manchester United, entendiéndose así "chiste" como algo irrisorio o débil (chistes $\mathrm{n}^{\mathrm{o}} 6$ y 7). En estos dos casos, la incongruencia se resuelve a través del mecanismo lógico del metarrazonamiento ${ }^{17}$. Por otro lado, hubo otros informantes que recrearon conversaciones inventadas en estilo directo, con sus correspondientes marcas tipográficas, como se observa en el ejemplo 2 y 12.

15 Chistes cortos basados en el juego de palabras muy típicos del habla inglesa.

$16 J K$ es un acrónimo en el argot inglés para decir Just Kidding.

17 El metachiste es un tipo de humor cuya incongruencia se genera al introducir el concepto de chiste dentro del propio chiste. Esta incongruencia requiere a su vez un metarrazonamiento para ser resuelta (cfr. Ruiz Gurillo 2012:50; Attardo, Hempelmann y Di Maio 2003: 16-17). 
Sin duda, la mayor dosis de creatividad la aportó un alumno de 15 años que diseñó sus propias viñetas cómicas. Se trata de unos dibujos que entrañan cierta agresividad, ya que el alumno hace eco de la muerte del tío de Spiderman en el cómic de Marvel y le parece gracioso el sufrimiento de superhéroe ${ }^{18}$. Vemos cómo además de emplear el estilo directo, se apoya también en el contenido gráfico propio de las viñetas cómicas.

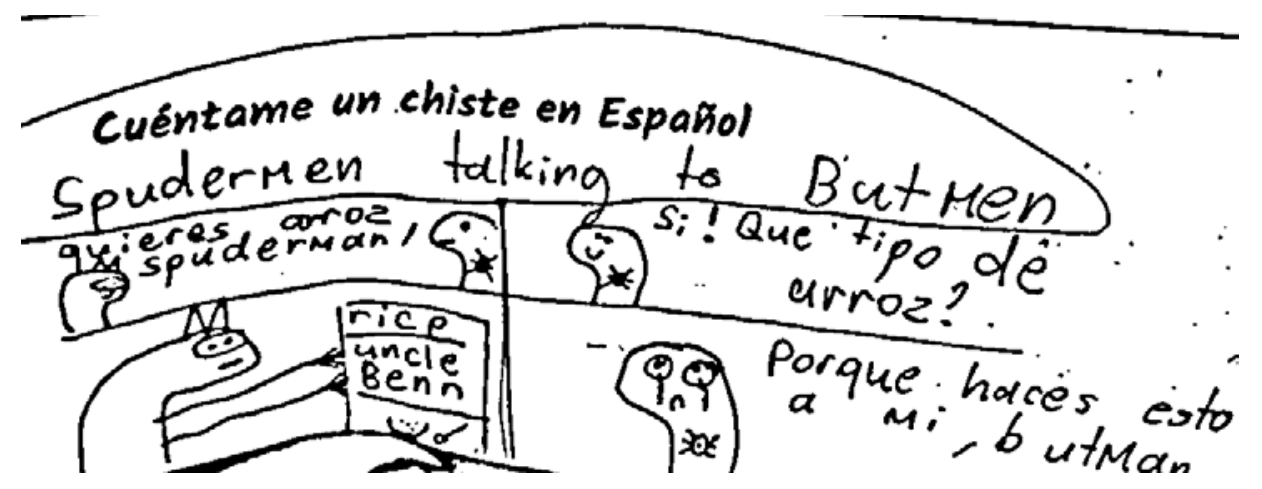

Figura 3. Viñeta cómica creada por alumno de 15 años con nivel B1.

Asimismo, las muestras de humor aquí expuestas corroboran la idea de Norrick (2007) de que en la producción del humor en L2/LE, los usuarios tienden a enfatizar su estatus de extranjero (ejemplos 2, 4, 6, 9 y 10) y/o hacen alusión a temas que para los nativos de esa lengua son tabú, ya que al no ser nativos no cargan con ningún lastre cultural y se sienten más liberados para hablar de ciertos temas (ejemplos 11, 14 y 16).

Por último, debemos señalar que la mayoría de los informantes que completaron satisfactoriamente esta pregunta llevaban en España más de 6 años y todos ellos cursaban el segundo ciclo de Educación Secundaria o Bachillerato. En este sentido, a fin de confirmar el efecto positivo de la inmersión lingüística en el desarrollo de la competencia humorística, creemos que sería pertinente realizar un estudio similar con adolescentes españoles que tengan un buen nivel de inglés como LE, a pesar de no estar en inmersión lingüística, para comprobar si ellos hubieran sido capaces de realizar este mismo ejercicio.

\section{CONCLUSIÓN}

Nuestro estudio sobre la apreciación y la producción del chiste en E/L2, realizado con 71 adolescentes extranjeros escolarizados en colegios internacionales de la Comunidad Valenciana y de la Región de Murcia, muestra las diferentes estrategias metapragmáticas empleadas por el oyente/lector extranjero para llegar a apreciar y producir humor en la lengua meta, en este caso el español. Además, se han analizado qué factores influyen en la evolución de la competencia humorística y comunicativa, examinando variables como el grado de inmersión lingüística, el tiempo de estancia en España, el nivel de lengua, y el género y la edad del alumno.

18 En este caso, el informante ha decidido crear una situación en la que Batman le gasta una broma pesada a Spiderman, al ofrecerle una marca de arroz que se llama como su tío fallecido. 
Así, los resultados obtenidos con los distintos instrumentos metodológicos confirman que la evolución de la competencia humorística en E/L2 y, por tanto, el desarrollo cognitivo de los procesos de apreciación y producción del humor está directamente relacionado con el progreso lingüístico, pragmático y social del alumnado. Asimismo, hemos observado cómo estos fenómenos se ven también afectados por las variables género, edad, tiempo de residencia en el país de la L2 y lengua materna. Por otro lado, esta investigación demuestra que la madurez metapragmática, relacionada con las habilidades humorísticas de los hablantes no nativos, depende en gran parte de los conocimientos lingüísticos y socioculturales del estudiante. Por ello, no podemos validar nuestra segunda hipótesis; dado que, si bien es cierto que el nivel de lengua y el contexto de inmersión lingüística plena son variables trascendentales, el tiempo en España, el sistema educativo y el contacto con la lengua fuera del aula son factores que también influyen en el proceso de la adquisición de la competencia metapragmática, lo que permite evolucionar desde la epipragmática a una conciencia metapragmática plena. Finalmente, el escaso número de respuestas en la tarea sobre la producción del humor confirmaría nuestra tercera hipótesis acerca de la mayor complejidad cognitiva de este proceso en comparación con la apreciación del humor. Se trataría de un salto que solo aquellos con mayor competencia metapragmática en el humor son capaces de dar.

Ahora bien, con tal de poder realizar afirmaciones de carácter genérico, este trabajo requiere de una ampliación de la muestra y de un análisis más exhaustivo de los datos. Por ejemplo, convendría realizar el mismo estudio con adolescentes extranjeros escolarizados en institutos públicos españoles en los que la inmersión lingüística es plena. Además, podríamos también extender el estudio a niñas y niños extranjeros, menores de 12 años, escolarizados en España, para así estudiar cómo se manifiesta la competencia epipragmática. De igual modo, necesitamos un análisis más profundo sobre la edad de nuestros participantes adolescentes, con el fin averiguar si las habilidades metapragmáticas mejoran en los últimos años de la adolescencia.

En definitiva, este estudio sobre la apreciación y producción del chiste en E/L2 demuestra que esta manifestación humorística es propicia para ser introducida en los materiales didácticos desde niveles iniciales. De hecho, como hemos visto con los resultados en $\$ 4.1$ y $\S 4.2$, el chiste es un género con el que se puede trabajar tanto la competencia como la producción humorística. No cabe duda de que muchas de las situaciones de humor fallido se podrían evitar si introdujéramos muestras de humor desde niveles iniciales en el aula. Su inclusión en el aula de E/L2 significaría un primer acercamiento al humor en la lengua meta y la evolución positiva de la competencia metapragmática del humor en la L2. Estamos convencidos de que el desarrollo de la competencia humorística comporta una mejora de la competencia comunicativa y, por tanto, de las habilidades sociales de nuestros estudiantes.

\section{Referencias bibliográficas}

Alvarado Ortega, M.B. (2012): "Una propuesta de estudio para el humor en la conversación coloquial”. En Estudios de Lingüística, 26, pp. 7-28.

Alvarado Ortega, M. B. (2014). "Humor y género: análisis de conversaciones entre mujeres". Feminismo/s, 24, pp. 17-40.

Andrews, T. G. (1943). "A factorial analysis of responses to the comic as a study in personality". The Journal of General Psychology, 28(2), pp. 209-224. 
Askildson, L. (2005). "Effects of humor in the language classroom: Humor as a pedagogical tool in theory and practice". Arizona Working Papers in SLAT, 12(1), pp. 45-61.

Attardo, S. (1994). Linguistic theories of humor. Berlin: Mouton de Gruyter.

Attardo, S. (2001). "Humor and irony in interaction: from mode adoption to failure of detection." In L. Anolli, R. Ciceri \& G. Riva (eds.): Say not to say: new perspectives on miscommunication Ámsterdan: IOS Press, pp. 166-186

Attardo, S., Hempelmann, C. F., \& Di Maio, S. (2003). "Script oppositions and logical mechanisms: Modeling incongruities and their resolutions". HUMOR: International Journal of Humor Research, 15(1), pp. 3-46.

Bell, N. D. (2005). Exploring L2 language play as an aid to SLL: A case study of humour in NS-NNS interaction. Applied Linguistics, 26(2), 192-218.

Bell, N. (2009). "Learning about and through humor in the second language classroom". Language Teaching Research, 13(3), pp. 241-258.

Bell, N, \& Attardo, S. (2010). "Failed humor: Issues in non-native speakers' appreciation and understanding of humor". Intercultural Pragmatics, 7(3), pp. 423-447.

Bell, N. (2011). "Humor scholarship and TESOL: Applying findings and establishing a research agenda”. TESOL Quarterly, 45(1), pp. 134-159.

Bell, N. (2013). "Responses to incomprehensible humor". Journal of Pragmatics ,57, pp. 176-189.

Bergson, H., Brereton, C. S. H., \& Rothwell, F. (1911). Laughter: An essay on the meaning of the comic. Macmillan.

Boxer, D., \& Cortés-Conde, F. (1997). "From bonding to biting: Conversational joking and identity display". Journal of Pragmatics, 27(3), 275-294.

Carrel, A. (1997). “Joke competence and humor competence". Humor 10(2), pp. 173-185.

Carbajal Carrera, B. (2013) "Grados de espontaneidad en el humor. Implicaciones del caso de la viñeta en el reconocimiento y apreciación de los mensajes humorísticos”. Pragmalingüística, (21), pp. 41-58.

Carretero-Dios, H. (2005). Sentido del humor: construcción de la escala de apreciación del humor (EAHU). Universidad de Granada. (Tesis doctoral disponible en https://hera.ugr.es/tesisugr/15472310.pdf)

Consejo de Europa (2002). Marco común europeo de referencia para las lenguas: aprendizaje, enseñanza y evaluación. $<\mathrm{http}: / /$ cvc.cervantes.es/obref/marco $>$. Ministerio de Educación, Cultura y Deporte - Instituto Cervantes - Anaya, 2003.

Crespo, N., Benítez, R., Pérez, L. (2010) "Conciencia metapragmática y la habilidad para producir narraciones escritas”, Revista Signos, 43 (73), pp. 179-209.

Davies, C.E. (2003). "How English-learners joke with native speakers: an interactional sociolinguistic perspective on humor as collaborative discourse across cultures". Journal of Pragmatics, 35, pp. $1361-85$.

Deneire, M. (1995). "Humor and foreign language teaching". Humor: International Journal of Humor Research.,8, pp. 285-298

Eysenck, H. J. (1942). "The appreciation of humour: An experimental and theoretical study". British Journal of Psychology, 32(4), 295-309.

Gombert, J. (1992): Metalinguistic development. Chicago: University of Chicago Press.

Gironzetti, E. (2013). Estudio pragmático experimental del humor gráfico en español e italiano y sus aplicaciones para la enseñanza de una LE/L2. Universidad de Alicante (Tesis doctoral disponible en http://hdl.handle.net/10045/35847)

Gironzetti, E., Attardo, S. \& Pickering, L. (2016). "Smiling, gaze and humor in Conversation" En Ruiz-Gurillo, L. (ed.): Metapragmatics of humor: Current Research Trends. Amsterdam: John Benjamins.

Gombert, J. (1992). Metalinguistic development. Chicago: University of Chicago Press.

Grice, H. P. (1975). "Logic and Conversation", en Cole, P. y J. Morgan (eds.): Syntax and Semantics, 3, New York, Academic Press, pp. 41-58 
Grupo GRIALE. (2011). ¿Estás de broma? 20 actividades para practicar la ironía en clase de ELE. Madrid, Edinumen.

Hay, J. (2000). "Functions of humor in the conversations of men and women". Journal of pragmatics, 32(6), pp. 709-742.

Hay, J. (2001). "The pragmatics of humour support". Humor: International Journal of Humor Research 14 (1), pp. 55-82.

Hess-Zimmermann. (2016). "Is this a joke? Metalinguistic reflections on verbal jokes during the school years". E-JourALL, EuroAmerican Journal of Applied Linguistics and Language, 3 (2), pp. 3-21

Instituto Cervantes (2006). Plan curricular del Instituto Cervantes. Madrid: Instituto Cervantes, Biblioteca Nueva.

Jefferson, G. (1984). "On the organization of laughter in talk about troubles". In J. M. Atkinson \& J. Heritage (Eds.) Structures of social action. Cambridge, England: Cambridge University Press, pp. 347-369.

Kotthoff, H. (2006). "Gender and humor: The state of the art." Journal of pragmatics, 38(1), pp. 4-25.

Lefcourt, H. M. (2001). Humor: The psychology of living buoyantly. Springer Science \& Business Media.

Norrick, N. (2007). "Interdiscourse humor: Contrast, merging, accommodation." Humor: International Journal of Humor Research, 20, 389-413. doi:10.1515/ HUMOR.2007.019.

McGhee, P. E. (2002) Understanding and Promoting the Development of Children's Humor. Dubuque, Kendall Hunt Publishing.

Martín Peris, E. (1991). "La didáctica de la comprensión auditiva”. revista Cable, 8, pp. 1-11.

Martín Peris, E., Cortés, M., \& Lopez, C. (2008). Diccionario de términos clave de ELE del CVC. Madrid: SGEL. Disponible en: http://cvc.cervantes.es/ensenanza/biblioteca_ele/diccio_ele/indice.htm

Martin, R. A., Puhlik-Doris, P., Larsen, G., Gray, J., \& Weir, K. (2003). "Individual differences in uses of humor and their relation to psychological well-being: Development of the Humor Styles Questionnaire". Journal of research in personality, 37(1), pp. 48-75.

Martin, R.A (2007). The Psychology of Humor: An Integrative Approach. Burlington: Elsevier Academic Press.

Orekoya, O.S.; E. S. Chan; M. P. Chik (2014) "Humor and Reading Motivation in Children: Does the Tickling Work?" International Journal of Education, 6:1, pp. 61-72.

Pastor Cesteros, S. (2004). Aprendizaje de segundas lenguas. Lingüística aplicada a la enseñanza de idiomas. Alicante: Publicaciones de la Universidad de Alicante

Raskin, V. (1985). Semantic Mechanisms of Humor. Reidel, Dordrecht

Reddington, E., \& Waring, H. Z. (2015). Understanding the sequential resources for doing humor in the language classroom. Humor, 28(1), 1-23.

Ruiz Gurillo, L. (2008): "El lugar de la ironía en la clase de español como lengua extranjera. Más allá del Marco y del Plan Curricular", RedELE, 14. Disponible en http://rua.ua.es/dspace/bitstream/10045/13076/1/Leonor_Ruiz_Lugar_ironia.pdf

Ruiz Gurillo, L. (2010): "Para una aproximación neogriceana a la ironía en español”, Revista Española de Lingüistica, 40/2, pp. 95-124.

Ruiz Gurillo, L. (2012). La lingüística del humor en español. Madrid: Arco Libros.

Ruiz Gurillo, L. \& Alvarado Ortega, M. B. (eds.) (2013a). Irony and Humor: from pragmatics to discourse. Amsterdam: John Benjamins.

Ruiz Gurillo, L. (2014): "Infiriendo el humor. Un modelo de análisis para el español”, Revista CLAC (Círculo de Lingüistica Aplicada a la Comunicación), 59, pp. 148-162. http://pendientedemigracion.ucm.es/info/circulo/no59/rgurillo.pdf

Ruiz Gurillo, L. (2015b): “¡Marte es divertido! Sobre humor, apreciación y emociones en niños”, Revista Pragmalingüística, 23, pp. 8-28. 
Ruiz Gurillo, L. (2016a). "Metapragmatics of humor: Variability, negotiability and adaptability in humorous monologues". En Ruiz-Gurillo, L. (ed.): Metapragmatics of humor: Current Research Trends. Amsterdam: John Benjamins, pp. 79-101 (doi 10.1075/ivitra.14.01int).

Ruiz Gurillo, L. (2016b): “Humor” en Gutiérrez-Rexach, J. (eds.): Enciclopedia de lingüística hispánica. London/NY, Routledge.

Sacks, H. (1974). "An analysis of the course of a joke's telling in conversation”. In R. Bauman \& J. Sherzer (Eds.), Explorations in the ethnography of speaking. London: Cambridge University Press, pp. 337-353

Shardakova, M. (2010). "How to be funny in a second language: Pragmatics of L2 humor". Mnemosynon. Studies on language and culture in the Russophone world: A collection of papers presented to Dan E. Davidson by his students and colleagues, pp. 288-310.

Schmitz, J.R. (2002). "Humor as a pedagogical tool in foreign language and translation courses". Humor: International Journal of Humor Research, 15(1), pp. 89-113.

Shively, R. L. (2013). "Learning to be funny in Spanish during study abroad: L2 humor development". The Modern Language Journal (97), pp. 930-946. (doi:10.1111/j.1540-4781.2013.12043.x)

Suls, J. M. (1972). "A two-stage model for the appreciation of jokes and cartoons: an information processing analysis", Goldsteins J. H. y P. E. McGhee (eds.): The Psychology of Humor. Nueva York: Academic Press, pp. 81- 100.

Timofeeva, L. (2014). "El humor verbal en niños de Educación Primaria: desarrollo de la conciencia metapragmática". Feminismo/s, (24), pp. 195-219.

Timofeeva, L. (2016). "Children using phraseology for humorous purposes” En Ruiz-Gurillo, L. (ed.): Metapragmatics of humor: Current Research Trends. Amsterdam: John Benjamins, pages 273-298 (DOI: 10.1075/ivitra.14.14tim)

Timofeeva, L. (2017). "Metapragmática del humor infantil”. Círculo de Lingüística Aplicada a la Comunicación, 70, pp. 5-19.

Verschueren, J. (1999). Understanding Pragmatics. London: Edward Arnold / New York: Oxford University Press.

Verschueren, J. (2000). "Notes on the role of metapragmatic awareness in language use", Pragmatics, 10:4, pp. 439-456.

Yip, J. A., \& Martin, R. A. (2006). "Sense of humor, emotional intelligence, and social competence". Journal of Research in Personality, 40(6), pp. 1202-1208.

Zadjman, A. (1995). "Humorous face-threatening acts: Humor as a strategy". Journal of Pragmatics. (23), pp. 325-339. 